\title{
PMA - Un psychanalyste éclaire de formidables enjeux
}

\section{Jean Martin}

Dr med., membre de la rédaction, ancien membre de la Commission nationale d'éthique

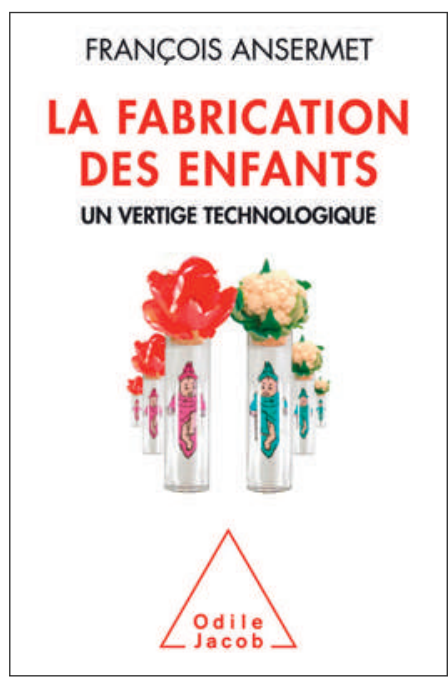

François Ansermet

La fabrication des enfants - Un vertige technologique

Paris: Ed. Odile Jacob; 2015.

256 pages. 23.90 EUR.

ISBN 978-2-738-13250-5

Les questions liées à la procréation médicalement assistée (PMA), et ses diverses formes qui se sont développées depuis la naissance en 1978 de Louise Brown, premier «bébé-éprouvette», sont complexes. La Commission nationale d'éthique a publié récemment un rapport à ce sujet [1]. Le Prof. François Ansermet, chef du service de psychiatrie de l'enfant et de l'adolescent aux Hôpitaux Universitaires de Genève publie un ouvrage substantiel sur ces sujets.

Venant d'un praticien de la psychanalyse, on pourrait imaginer que ce livre ne soit pas aisément accessible. Ce n'est pas le cas; même si le caractère impensable, irreprésentable ou indicible de plusieurs de nos interrogations est discuté à réitérées reprises, le propos est concret, bien écrit, avec de nombreuses notations et vignettes cliniques. Un appareil de notes et une bibliographie fournissent de nombreuses références complémentaires.
Les PMA sont discutées sur le décor de l'évolution générale de notre société, marquée par la possibilité de poursuivre tant d'objectifs inimaginables pour nos prédécesseurs. "Concevoir un enfant peut aussi viser à annuler le temps. On rejoint la pente prise par le monde contemporain, où l'on cherche à atteindre une jouissance du tout, tout de suite: une jouissance qui est revendiquée comme un droit.»

Constamment, l'auteur met en garde contre les explications ou conclusions simples, univoques. Il insiste sur le fait que dans ce domaine comme en général en biologie, en médecine et dans la vie, il y a coexistence, co-action, d'éléments du registre de la nature et d'autres de la culture; développements et évènements dans nos vies sont de causalité multifactorielle (penser à l'épigénétique et à l'influence du milieu - dès avant la naissance). "Toutes les démarches biotechnologiques réalisent une connexion nouvelle entre le vivant et la culture.» L'ouvrage compte quatre parties, dont trois incluent «Vertiges» dans leur titre: vertiges de l'origine, de la différence, du destin; le quatrième est «Vers une clinique du devenir».

\section{Des découplements inédits - On avance vers l'inconnu}

Dans l'introduction: «Savoir comment on fait un enfant reste un thème inabordé, hors du dicible [...] Les biotechnologies permettent d'intervenir sur la nature, de la modifier, sans qu'on connaisse pour autant les conséquences de ce qu'on a rendu possible. On crée une réalité différente qui fait buter sur l'inconnu.» L'évolution est caractérisée par le découplement, la séparation entre des éléments qui auparavant étaient forcément liés: ainsi sexualité et procréation, procréation et filiation (sauf le cas de l'adoption). Des situations nouvelles sont créées par les dons de gamètes ou d'embryon, ainsi que par la grossesse pour autrui (GPA - non autorisée en Suisse à ce stade quoique des interventions parlementaires s'en préoccupent). Ansermet évoque la faisabilité de la transplantation d'utérus, qui porte à six le nombre de personnes pouvant être impliquées dans la naissance d'un enfant: donneuse 


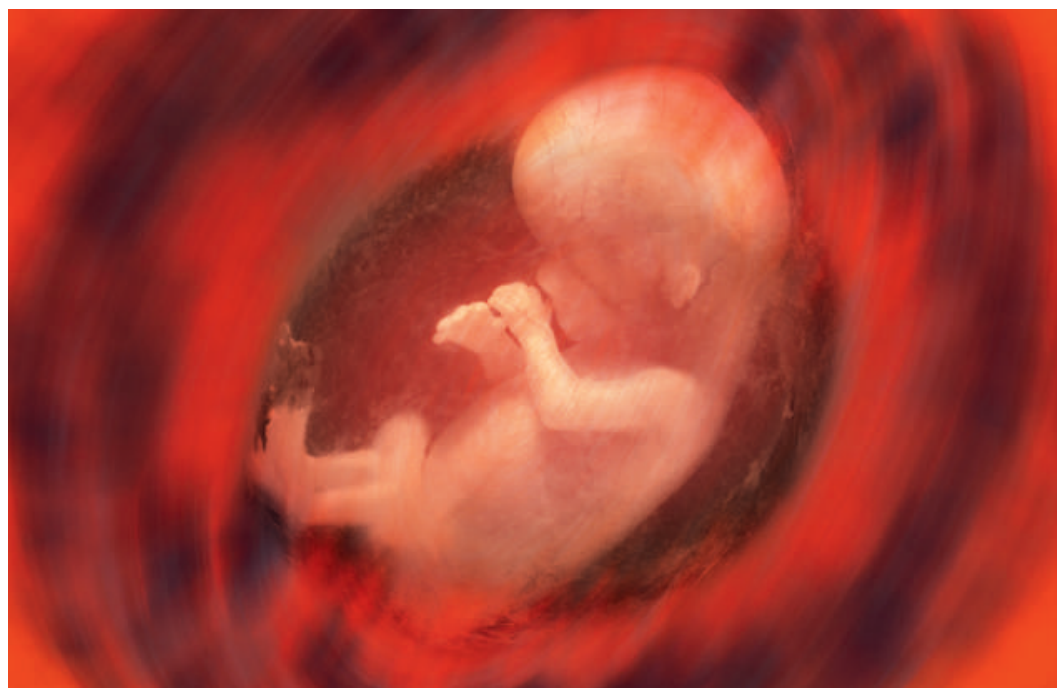

Un enfant a été créé et se développe: vertige de l'humanité - et parfois de la technologie.

d'ovule, donneur de sperme, donneuse d'utérus, mère porteuse, et les deux parents sociaux/légaux (cas échéant couple gay - un chapitre est consacré à la procréation homosexuelle).

Les cas qui rendent perplexe fourmillent. "Qu'implique ainsi le don d'utérus, lorsqu'une mère donne son utérus à sa fille [transplantation] pour que celui-ci lui soit greffé afin qu'elle puisse porter un enfant dans l'utérus qui l'a elle-même portée? On mesure à quel point une greffe d'utérus peut être aussi une greffe d'imaginaire pour le père/mari, avoir une partie de sa belle-mère à l'intérieur du corps de sa femme pour porter son enfant peut être lourd en termes de représentation.»

Sur la grossesse pour autrui: «Quant à la GPA, pourrat-on un jour savoir de quel ventre maternel on provient? Pourra-t-on suivre à la trace les empreintes épigénétiques? Les disjonctions introduites par les possibilités des biotechnologies entre le génétique, d'une part, le juridique, le social ou le psychique vont impliquer la nécessité de construire une dialectique nouvelle entre toutes ces dimensions. Notons que l'auteur est défavorable à ce qu'on dénomme "père biologique» le donneur de sperme. Les statut et rôle de père vont évidemment bien au-delà de la mise à disposition de cellules.

\section{DPI - et plus loin «bébé-médicament»?}

S'agissant de l'enjeu politique suisse actuel du diagnostic préimplantatoire, Ansermet y est favorable. Il s'exprime aussi sur ce qu'on appelle «bébé-médicament», ou «bébé-sauveur» (conçu aussi pour pouvoir aider un frère ou une sœur malade déjà né). Sans exclure de possibles difficultés au sein de la fratrie, il se dit ouvert à la problématique (c'est ma propre position, j'apprécie qu'un psychanalyste puisse s'y rallier). N.B.: On dit aussi en France «bébé du double espoir»: «espoir de faire naître un enfant sain, qui ne soit pas porteur de la maladie génétique et, deuxième espoir, que cet enfant conçu avec le DPI puisse aider au traitement d'un enfant malade [...] Qu'un enfant soit conçu pour un projet ne vient pas l'aliéner dans ses autres potentialités. Finalement, on fait toujours aussi un enfant pour quelque chose d'autre, consciemment ou inconsciemment [...] L'enfant du double espoir est le créateur d'un devenir en ayant permis à un frère ou une sœur une vie meilleure: on peut imaginer pire destin pour un sujet à sa naissance!»

Cela change agréablement des «scénarios à la Frankenstein» brandis par certains opposants. Au reste, rappelons que ce dernier sujet n'est pas à l'ordre du jour en Suisse à ce stade. En 2016, nous voterons sur un texte légal qui exclut spécifiquement le «bébé-médicament».

\section{Cas particuliers en question}

Par exemple, les possibilités ouvertes par la cryoconservation des gamètes et embryons, dont la situation de jumeaux (embryons fécondés/obtenus en même temps) qui naîtraient à des années voire une génération de distance parce que l'un des deux a été cryoconservé. Question aussi de ce qu'on appelle la cryo-auto-conservation sociétale des ovules - chez des femmes qui veulent repousser, pour des raisons professionnelles notamment, le moment d'être enceintes mais veulent l'être avec des ovules jeunes...

Est déjà étudiée l'hypothèse de l'obtention de gamètes (des deux sexes) à partir de cellules-souches reprogrammées: «Demain on pourra peut-être ajouter une autre figure de la mère, beaucoup plus abstraite: celle de cellules-souches de la peau, reprogrammées pour devenir des gamètes. Une cellule souche totipotente viendrait ainsi se substituer aux protagonistes de la procréation» (qui jusqu'ici sont des personnes physiques identifiables).

Bien que le clonage reproductif (une parthénogenèse) ne soit guère abordé dans le cadre PMA, Ansermet consacre un utile chapitre à ce sujet et aux fantasmes associés. Il discute l'association de l'idée de clonage avec celle d'immortalité, tout en la disqualifiant: le clone ne saurait être la même personne prolongée à l'identique.

«Toutes les PMA ont en perspective un contournement de la sexualité qui est au fondement des théories sexuelles infantiles, que le clonage réalise de façon radicale, contournant non seulement les pratiques sexuelles mais la procréation entre deux gamètes ellemême. 


\section{Prédiction, prévention}

Dans la partie «Vertiges du destin» est traitée en détail la problématique de la prédiction, liée aux choix multiples que la PMA pourra(it) permettre. «Le Meilleur des mondes de Huxley est-il au bout de la PMA?», demande-t-il. Mais il relativise le caractère vu parfois comme totalement déterminant de ces potentialités. «Il s'agit de bien saisir le côté illusoire de telles perspectives, en rappelant que l'instabilité génétique, l'impact des facteurs épigénétiques et la plasticité font aller audelà d'une telle vision déterministe. C'est ainsi que la prédiction ne peut prendre la place du hasard.»

\section{Dans l'activité clinique - Besoin d'espaces ouverts}

«Qu'un enfant ait été conçu par PMA ne préjuge en rien de l'avenir. Il s'agit d'aller contre la tendance de voir dans les PMA la cause à tout faire de ce que devient le sujet.» "L'enjeu est bel et bien de ne pas faire un destin des conditions de sa propre procréation.»

"S'il y a une tâche pour le clinicien, c'est d'aider les parents à se dégager des constructions imaginaires qui les encombrent, suite aux contraintes du traitement de la stérilité. Et, du côté de l'enfant, de lui ouvrir un espace d'imprévisibilité. Au-delà du fait d'être issu d'une PMA, de l'aider à s'ouvrir au champ des possibles, afin qu'il puisse devenir l'auteur et l'acteur de son propre devenir.»

«Peut-être faut-il revenir plutôt au cas par cas de la clinique. Et se dire qu'il n'y a finalement d'éthique que du particulier.» Vaste thématique! Devrait-on/pourrait-on renoncer à établir des règles s'appliquant à tous sur la pratique des PMA?

\section{Un avenir complexe qu'il faut assumer, au carrefour des valeurs et des techniques}

"La médecine génétique contemporaine nous convoque à une nouvelle époque de la tragédie. Les forces supérieures qui manipulent les hommes ne sont plus les dieux mais les gènes.» «Le point de vertige tient au fait qu'avec ces technologies, on touche à la différence des sexes et à celle des générations, les deux différentiels sur lesquels repose l'ordre symbolique. On doit faire face au vide, ou à des représentations mises en crise.» «Le fantasme peut prendre lui-même une place dans la démarche scientifique. Plus particulièrement comme promoteur de ses inventions technologiques. Le fantasme n'est donc pas antinomique de la science. Il en est même plutôt corrélatif.»
«Les nouveaux modes d'origine rendus possibles par les PMA peuvent être connectés à des désirs qui sont revendiqués comme des droits. Et la loi est toujours en retard sur la science, jusqu'à produire des situations où le désir offense la loi.» "Au-delà du débat sur la possibilité d'une procréation homosexuelle, ce type de perspective pose la question connexe très importante d'une tendance à la médicalisation de la procréation, qui pourrait être de plus en plus banalisée pour devenir peut-être finalement un passage obligé.» La PMA qui pourrait devenir un passage obligé? Vers la désaffection généralisée de la manière de faire des enfants qui a été la nôtre jusqu'ici? Certains adeptes de sciencefiction l'imaginent.

«Les indications sociétales des PMA font aller vers un monde nouveau. On doit faire face à de nouvelles façons de donner la vie, de la concevoir, dans tous les sens du terme. L'inconcevable de la conception d'un enfant reste au coeur du questionnement. Il s'agit de ne pas tomber dans les pièges du biocatastrophisme ni de se laisser aller sur la pente d'une tentation conservatrice.»

Le tableau que brosse François Ansermet intéressera tous ceux que préoccupent les défis, sociaux et sociétaux, philosophiques et bioéthiques, médicaux, juridiques, pratiques, que lancent les avancées de la biomédecine en général et des PMA en particulier. Ses analyses et commentaires sont bien informés, équilibrés à mon sens, y compris en laissant/donnant à chacun, dans sa singularité, sa responsabilités propre. Un exposé vaste et critique, non-pessimiste-mais il se peut que les "pessimistes» trouvent qu'il n'est pas assez alarmé.

Une mise en garde toutefois: «On voudrait échapper aux incertitudes qu'impose la nature, aller vers un nouveau programme de l'humain. Eviter les maladies, mobiliser toutes les potentialités. Jusqu'à l'excès, jusqu'à l'idée d'échapper à la finitude, à la mort, de tout pouvoir contrôler dès avant la conception. [...] A trop forcer la réalité, on peut la faire délirer. Un des caractéristiques du débat autour des biotechnologies est de ne plus savoir où est la limite, où la mettre [...] Ce qui est rendu possible par le fait des technologies ne doit pas nécessairement avoir lieu.»

\section{Références}

1 Commission nationale d'éthique pour la médecine humaine (CNE-NEK). La procréation médicalement assistée-Considérations éthiques et propositions pour l'avenir. Prise de position No. 22/2013. Berne, décembre 2013 (disponible aussi en allemand, italien et anglais).

\section{Crédit photo}

(c) Steve Allen | Dreamstime.com 